\title{
Câncer de estômago: fatores de risco
}

\author{
Stomach cancer: risk factors
}

Anna Valéria de Britto 1

\footnotetext{
1 Departamento de Medicina Preventiva e Social, Faculdade de Ciências Médicas, Universidade Estadual de Campinas. C. P. 6111 , Campinas, SP 13081-970, Brasil.
}

Abstract For the last fifty years, stomach cancer has become less important in terms of morbidity and mortality in devel oped countries, but the same pattern has not occurred in Brazil. The main risk factors for this neoplasm are certain di et patterns that became evident through epidemiological studies in various population groups. After the carcinogenic effects of nitrosamines and the anti-oxidant activity of vitamin $\mathrm{C}$ were discovered, some of the associations between stomach cancer and diet were partially understood. After the description of Helicobacter pylori in 1983 and the evidence of the relationship between this bacteria and certain stomach diseases, new el ements were added to the knowledge about the devel opment of this neoplasm. Current knowledge, al beit incomplete, provides interesting prospects for the prevention and early diagnosis of stomach cancer.

Key words Cancer; Stomach Cancer; Risk Factors to Stomach Cancer; Epi demi ology

Resumo Há cinqüenta anos o câncer de estômago vem perdendo a importância em termos de morbi-mortalidade em países consi derados de primei ro mundo. Isso não ocorre no Brasil. Os princi pais fatores de risco evi denciados a partir de estudos epi demi ológi cos em várias populações do mundo e associados a essa neoplasia são al guns padrões de di eta. Com a descoberta do papel carcinogênico das nitrosaminas e do potencial anti-oxidante da vitamina $C$, algumas das associ ações entre câncer gástrico e padrões de di eta passaram a ser parcialmente entendidas. Com a descrição da Hel i cobacter pylori em 1983 e as evidências da relação dessa bactéria com certos processos patológi cos do estômago, al guns precursores do câncer gástri co, novos el ementos foram agregados ao processo fisiopatológi co dessa entidade. O conhecimento hoje adquirido sobre a fi si opatol ogia do câncer gástrico, mesmo que parcial, fornece perspectivas esti mulantes para prevenção e diagnóstico precoce.

Palavras-chave Câncer; Câncer de Estômago; Fatores de Risco para Câncer de Estômago; Epi demiologia 
O câncer de estômago (CE) no Brasil ainda é importante causa de morbi-mortalidade em regiões mais desenvolvidas como o Sul e Sudeste, assim como nas de menor desenvolvimento. Os coeficientes de incidência padronizados pela população mundial, no sexo masculino, variam, por cem mil habitantes, de 51,6 em Belém, em 1989, a 22,4 em Goiânia, em 1991. Os coeficientes padronizados de mortalidade, no sexo masculino, variam, por cem mil habitantes, de 37,3 em Belém, em 1988, a 12,5 em Goiânia, em 1991 (MS, 1995).

No Brasil, parece que o decréscimo da mortalidade por essa neoplasia em análises de algumas décadas, como pode ser apreciado em outro artigo dessa revista (Latorre, 1997), não caracteriza a situação vista em outros países, que apresentaram importante redução da mortalidade por CE nos últimos cinqüenta anos, caracterizando um "triunfo não planejado" (Howson et al., 1986).

Na literatura nacional, pouco pode ser encontrado sobre trabalhos que avaliam a prevalência de fatores de risco para CE no Brasil. Na literatura internacional, o que pode hoje ser sistematizado sobre o processo de carcinogênese gástrica vem sendo determinado através de evidências de estudos clínicos, epidemiológicos e experimentais em animais.

Sabe-se que o CE surge com as alterações da mucosa gástrica, que sob ação de vários fatores, adquire um fenótipo progressivamente regressivo, com substituição das células normais por aquelas que existem naturalmente no intestino (delgado e, posteriormente, grosso), no sentido inverso ao que ocorre durante o desenvolvimento fetal. Esse processo de transmutação da mucosa gástrica, em parte conhecido, ocorre a longo prazo e sugere que os fatores de risco para CE atuam desde a mais tenra idade e por muito tempo.

A discussão que se segue sobre o CE do tipo intestinal (classificação segundo Lauren, 1965) baseia-se nas evidências provenientes de estudos epidemiológicos e experimentais em animais e no modelo de carcinogênese proposto por Pelayo Correa (Correa, 1988). Segundo esse autor, as fases que antecedem o CE formam um continuum, do epitélio gástrico normal a gastrite, que se torna crônica e evolui para atrófica com perda de glândulas, seguida de metaplasia do tipo intestino delgado e depois colônica, displasia e câncer. Acompanhando essas alterações, há diminuição da secreção ácida, síntese anormal de mucinas ácidas e excessívos níveis de nitrato e nitrito na cavidade gástrica.

Há bastante tempo a ocorrência do CE vem sendo associada à exposição a fatores relacio- nados com a dieta. Estudos epidemiológicos sobre os padrões de ocorrência e óbito por CE em populações de migrantes (Haenszel, 1961; Haenszel \& Kurihara, 1968; Haenszel et al., 1972; Correa et al., 1973; McMichael et al., 1980; Kolonel et al., 1981, 1983; Miller, 1982; Rosen Waike, 1984; Correa et al., 1985; Jedrychowski et al., 1986; Hu et al., 1988) vêm reforçando a idéia da associação entre essa neoplasia e alguns fatores encontrados em certos padrões de dieta, dentre eles o sal, hoje imputado como um fator que lesa a mucosa gástrica facilitando a ação de agentes genotóxicos. Evidências experimentais foram obtidas com ratos que receberam dietas ricas em sal, comprovando que este leva à atrofia gástrica (Capoferro \& Torgensen, 1974; Kodama et al., 1984), além de estar fortemente associado a metaplasia, facilitar a carcinogênese no estômago na presença de outros carcinógenos (Tatematsu et al., 1975; Ohgaki \& Kato, 1984; Takahashi et al., 1984; Correa, 1988) e aumentar a absorção dos hidrocarbonetos policíclicos aromáticos (Capoferro \& Torgensen, 1974), que são substâncias carcinogênicas para animais e, provavelmente, para seres humanos.

Talvez, boa parte do decréscimo da incidência do CE há algumas décadas tenha se devido à abolição do uso do sal para conservar alimentos, pois, segundo o sistema de carcinogênese aqui adotado, tal uso é um dos fatores que atuam nos primórdios do processo de alteração da mucosa gástrica (Ohgaki \& Kato, 1984; Takahashi et al., 1984; Correa, 1988).

Mais recentemente, começou a ser reconhecido o potencial carcinogênico das nitrosaminas e nitrosamidas, genericamente chamadas de N-compostos (NOC), substâncias formadas com a interação entre um grupo de nitrogênio secundário (que pode ser uma amina ou amida, uma alquil-uréia ou um anel peptídeo) e um nitrito. A evidência de que as populações de maior risco para o CE apresentavam dietas ricas em substratos para o processo de produção endógena de NOC desencadeou o interesse na avaliação dessas substâncias na gênese do CE.

Foi demonstrado por Sander \& Schweinsberg (1972) que as aminas e nitritos ingeridos na dieta podiam reagir in vivo para produzir $\mathrm{N}$ nitrosaminas e N-nitrosamidas. Mirvish et al. (1972) mostraram que o ascorbato reduz a formação de tumores em animais quando seguido pela alimentação de nitritos e aminas, provavelmente por inibir a formação de NOC in vivo. Em revisão posterior, o mesmo autor reforça esses pressupostos (Mirvish, 1994).

Esses achados foram consistentes com trabalhos epidemiológicos que evidenciavam o 
papel de dietas ricas em precursores dos Ncompostos como fatores de risco para CE (Armijo \& Coulson, 1975; Cuello et al., 1976; Haenszel et al., 1976; Correa et al., 1979, Tannenbaum et al., 1979; Fraser et al., 1980; Armijo et al., 1981) e de dietas ricas em alimentos que contêm vitamina $C$, um potente anti-oxidante, como protetoras contra o CE (Weisburger, 1985a).

Entre os alimentos apontados como os que elevam o risco, estão principalmente os defumados, carnes curadas, peixes secos e outros alimentos conservados em sal. Entre os que associam-se a baixo risco, encontram-se as frutas e vegetais. Estes, apesar de apresentarem elevada concentração de nitritos, talvez tenham efeito protetor pela presença da vitamina C. Alguns trabalhos indicam um efeito protetor de outros anti-oxidantes, como as vitaminas A e E (Graham, 1984; Risch et al., 1985; Stehr et al., 1985; Weisburger, 1985b; Bartsch et al., 1988), mas os resultados não são conclusivos.

Nos estudos epidemiológicos envolvendo os NOC, a diferenciação da produção endógena da que ocorre a partir da ingestão de precursores é importante para a determinação de fatores que possam estar contribuindo para o aumento do risco para CE e que não estejam relacionados à dieta. $\mathrm{O}$ teste NPRO (N-nitrosoprolina), para quantificar a nitrosação endógena em humanos in vivo descrito por Ohshima $\&$ Barstch (1981), constitui uma estimativa indireta do processo de nitrosação endógena. Com ele observou-se que, em certos indivíduos, não houve bloqueio da produção de compostos nitrosos pela vitamina $C$, o que reforça a hipótese de que eles são produzidos em sítios onde não há atuação desse bloqueador, e que esse dado pode ser importante na gênese do CE.

A consi deração desse fato nos estudos epidemiológicos é importante, pois as disparidades dos resultados encontrados sobre a associação entre CE e consumo de elevada concentração de nitritos pela dieta pode ser explicada, pelo menos em parte, pela dificuldade de controle das concentrações endógenas de NOC que surgem de fontes diferentes da dieta. Esse é um desafio a ser superado nas avaliações específicas sobre a importância de dietas ricas em precursores de NOC na gênese do CE.

Em relação aos carboidratos, parece que, se eles têm algum efeito na gênese do $C E$, esse é mais importante em etapas precoces da vida (Howson et al., 1986). Sabe-se que há uma importante tendência de dietas ricas em carboidratos serem pobres em proteína e gorduras. Esse fato pode determinar vieses nos estudos epidemiológicos que tentam estabelecer a importância da associação entre carboidratos e $C E$, pois a baixa ingesta protéica parece interferir na "qualidade" do suco gástrico como barreira a agentes tóxicos.

Quanto ao tabagismo, alguns estudos prospectivos mostram discreto risco aumentado entre os fumantes (Nomura et al., 1990; Kneller et al., 1991). Entre os estudos não prospectivos, alguns apontam aumento do risco (Hu et al., 1988; Risch et al., 1985) e outros não (Jedrychowsky et al., 1986; Lavecchia et al., 1987).

Considerando-se o consumo de álcool, alguns trabal hos demonstram risco aumentado para CE (Hoey et al., 1981; Jedrychowsky et al., 1986). No entanto, a maior parte dos estudos, coorte ou caso-controle, não demonstra o mesmo.

A partir de 1983, com a descrição das bactérias Gram-negativas flageladas e espiraladas por Warren \& Marshall (1983), denominadas Helicobacter pylori (HP), vários estudos começaram a ser feitos no sentido de determinar a patogenicidade desses microorganismos e suas relações com distúrbios gástricos, dentre eles o CE.

A associação da HP com gastrite crônica tipo $B$ vem sendo confirmada por inúmeros estudos em diferentes regiões do mundo. Sabese também que a gastrite crônica tipo $B$ relaciona-se com o CE, sendo um precursor desse na escala de evolução dessa patologia.

As semelhanças entre a epidemiologia do CE e HP, dentre elas a forte correlação positiva entre a alta prevalência dessa bactéria e a elevada incidência de CE em diversas populações do mundo, sugerem que possa existir uma relação causal, se bem que não suficiente, entre essas duas entidades.

Existem três trabalhos do tipo caso-controle (Forman et al., 1991; Nomura et al., 1991; Parsonnet et al., 1991), por meio dos quais foi demonstrada a presença de anticorpos contra HP em soro de indivíduos que posteriormente apresentaram o CE. Pelo desenho desses estudos, eles reforçaram a hipótese de associação causal entre HP e CE. Os resultados de outro trabalho, também tipo caso-controle, sugerem que a infecção por HP é um fator de risco independente para CE (Hansson et al., 1993).

Outros trabalhos, do tipo correlacionais, demonstram associações entre a prevalência da HP e mortalidade por CE (Forman et al., 1990), incidência de CE (ESG, 1993) ou risco para esse câncer (Correa et al., 1990). Geralmente estudos desse tipo são de difícil interpretação, possuem vieses cujo controle não é fácil e não evidenciam associação causal. 
O tipo de transmissão da HP não está estabelecido e cogita-se que podem existir múltiplas vias de transmissão (Goodman \& Correa, 1995). Parece que a contaminação se dá em fases precoces da vida, durante a infância, nas populações de países não desenvolvidos, onde sua prevalência é alta. Nesses, a prevalência da infecção em crianças com dez anos de idade pode ser de até 50\% (Marshall, 1994; Nightingale \& Gruber, 1994). O aparecimento de anticorpos contra HP ocorre em idades mais tardias nas populações onde há menor prevalência da HP e menor incidência do CE. Uma vez ocorrida a infecção, parece não haver resolução espôntanea e ela permanece oligo-assintomática por anos, levando à gastrite crônica e, às vezes, à gastrite crônica atrófica.

A HP parece se adaptar facilmente ao ambiente hostil do estômago, e há evidências de que, dentre outros danos, ela provoca o bloqueio do mecanismo natural da mucosa gástrica de concentrar e secretar o ácido ascórbico para o lúmen do estômago (Sobala et al., 1989; Taylor \& Blaser, 1991), além de aumentar a taxa de proliferação do epitélio gástrico e reduzir o nitrato a nitrito, o que é visto em algumas espécies de HP (Marshall, 1994).

\section{Conclusões}

O modelo de carcinogênese gástrica citado nesse trabalho decorreu de evidências que vêm sendo obtidas por meio de trabal hos clínicos, epidemiológicos e experimentais em animais. No entanto, apenas algumas das peças de um grande quebra-cabeça foram identificadas.

Os trabalhos epidemiológicos são consistentes na demonstração da relação de risco aumentado para o CE e consumo de dietas ricas em sal, e estudos experimentais confirmam os efeitos danosos deste para a mucosa gástrica.

A exposição aos NOC causada pela dieta ou devido a síntese endógena parece ser importante na gênese do CE. Esses compostos estão associados a mutações das células gástricas já alteradas pela gastrite atrófica crônica (Correa, 1992). No entanto, o processo de nitrosação em humanos é extremamente complexo e sua dinâmica, parcialmente conhecida. Apesar da evidência da importância de seu papel no desenvolvimento do CE, muito ainda é obscuro. As vitaminas $C$ e $E$ inibem a formação dos NOC no estômago (Mirvish, 1983), no entanto os resultados de estudos epidemiológicos são inconsistentes na demonstração do papel da vitamina $\mathrm{E}$ como efeito protetor contra o CE. Em relação à vitamina $\mathrm{C}$ e carotenóides, diversos estudos, alguns já citados nesse texto, vêm mostrando resultados coerentes quanto à associação negativa entre o elevado consumo desses elementos e CE em populações etnicamente distintas.

Apesar de alguns estudos apontarem risco aumentado para o CE entre fumantes e consumidores de álcool, são controversas essas associações.

Quanto à relação da HP e CE, muitas questões estão em aberto e parece que a infecção por essa bactéria é um fator contribuinte, mas não suficiente para o processo carcinogênico gástrico. A elevada prevalência dessa bactéria, de $80 \%$ em populações adultas de áreas de alta incidência e de $50 \%$ nas de baixa, indica que somente uma pequena parte desses casos evolui para CE. Também existem populações que, apesar da elevada prevalência de HP, apresentam baixa incidência de CE (Correa et al., 1990; Forman et al., 1990; Nomura et al., 1991; ESG, 1993; Hansson et al., 1993; Marshall, 1994; Nightingale \& Gruber, 1994). Esse fato aponta para a possibilidade de que as cepas de HP têm diferente potencial carcinogênico e, portanto, podem ser diferentes quanto ao aspecto antigênico (Sobala, 1991). Parece, então, que é importante validar testes sorológicos que se baseiam nesses pressupostos para que sejam usados em trabalhos epidemiológicos, no sentido de estimar de forma mais consistente os riscos (Megraud et al., 1989; Forman, 1991; Sobala, 1991; Bodhidatta et al., 1993; Muñoz, 1994).

Os odds ratio dos estudos de casos-controle aninhados (Forman et al., 1991; Nomura et al., 1991; Parsonnet et al., 1991), que variam de 2,7 a 6,0, sugerem a possibilidade de diminuição da incidência de CE com o controle da infecção pela HP, entre $35 \%$ a $60 \%$, baseada no risco atribuível estimado.

Esses fatos abrem perspectivas de intervenções sob o ponto de vista da Saúde Pública, seja interferindo nos padrões de dieta das populações de elevado risco para CE ou prevenindo a infecção por HP. Além disso, as alterações da mucosa gástrica nas etapas anteriores ao desenvolvimento do CE dão origem a marcadores pré-tumorais, como as mucinas sulfatadas e pepsinogênios do tipo I ell, que possibilitam a intervenção no sentido de realizar diagnósticos mais precoces (Miki et al., 1993; Correa, 1992), melhorando o prognóstico dessa patologia.

Sob o ponto de vista da epidemiologia, a maior parte do conhecimento sobre a relação do CE e dieta provém de estudos do tipo casocontrole, e pouco se conhece sobre a interação entre padrões dietéticos e infecção pela Helico- 
bacter pylori. Segundo os autores de extensa revisão recente sobre CE e dieta (Kono \& Hirohata, 1996), hoje se impõe aos epidemiologistas o desafio de elaborar estudos de coorte com levantamento detalhado e quantificado sobre dieta e micronutrientes, a fim de aprimorar o conhecimento sobre a relação desses fatores e CE.

\section{Referências}

ARMIJO, R. \& COULSON, A. H., 1975. Epidemiology of stomach cancer in Chile: the role of nitrogen fertilizers. International Journal of Epidemiology, 4:301-309.

ARMIJO, R.; ORELLANA, M. \& MEDINA, E., 1981. Epidemiology of gastric cancer in Chile. Case-control study. International Journal of Epidemiology, 10:53-56.

BARTSCH, H.; OHSHIMA, H. \& PIGNATELLI, B., 1988. Inibitors of endogenous nitrosation mechanisms and implications in human cancer prevention. Mutation Research, 202:307-324.

BODHIDATTA, L.; HOGE, C. M.; CHURNRATANAKUE, S.; NIRDNOY, W.; SAMPATHANUKUL, P.; TUNGTAEM, C.; RAKTHAM, S.; SMITH, C. D. \& ESCHEVERRIA, P., 1993. Diagnosis of Helicobacter pylori infection in a developing country: comparison of two ELISAs and a seroprevalence study. Journal of Infectious Diseases, 168:15491553.

CAPOFERRO, R. \& TORGENSEN, O., 1974. The effect of hypertonic saline on the uptake of tritiated 7 , 12 dimethylben(a)anthracene by the gastric mucosa. Scandinavian Journal of Gastroenterology, 9:343-349.

CORREA, P.; SASANO, N. \& STERM MERMANN, N., 1973. Pathology of gastric carcinoma in Japanese populations: comparison between Myiagi prefecture, Japan, Hawaii. Journal of the National Cancer Institute, 51:1449-1459.

CORREA, P.; CUELLO, C. \& GORDILLO, G., 1979. The gastric micro environment in populations at high risk to stomach cancer. National Cancer Institute Monography, 53:167-170.

CORREA, P.; FONTHAM, E.; PICKLE, L. W.; CHEN, V.; LIN, Y. \& HAENSZEL, W., 1985. Dietary determinants of gastric cancer in south Louisiana inhabitants. Journal of National Cancer Institute, 75: 645-653.

CORREA, P., 1988. A human model of gastric carcinogenesis. Cancer Research, 48:3554-3560.

CORREA, P.; FOX, J.; FONTHAM, E.; RUIZ, B.; LIN, Y.; ZAVALA, D.; TAYLOR, N.; MACKINLEY, D.; LIMA, E.; PORTILLA, H. \& ZARAMA, G., 1990. Helicobacter pylori and gastric carcinoma. Cancer, 66:25692574.
CORREA, P., 1992. Human gastric carcinogenesis: a multistep and multifactorial process. I American Cancer Society Award Lecture on Cancer Epidemiology and Prevention. Cancer Research, 52: 6735-6740.

CUELLO, C.; CORREA, P. \& HAENSZEL, W., 1976. Gastric cancer in Colombia: cancer risk and suspected environmental agents. Journal of the National Cancer Institute, 57:1015-1020.

ESG (EUROGAST STUDY GROUP), 1993. An International association between Helicobacter pylori infection and gastric cancer. Lancet, 341:1359-1362.

FORMAN, D.; SITAS, F.; NEWELL, D. G.; STACEY, A. R.; BOREHAM, J.; PETO, R.; CAM PBELL, T. C.; LI, J . \& CHEN, J., 1990. Geographic association of Helicobacter pylori antibody prevalence and gastric cancer mortality in rural China. International Journal of Cancer, 46:608-611.

FORM AN, D.; NEWELL, D. G.; FULLERTON, F.; YARNELL, J. W. G., STACEY, A. R.; WALD, N. \& SITAS, F., 1991. Association between infection with Helicobacter pylori and risk of gastric cancer: evidence from a prospective investigation. British Medical Journal, 302:1302-1305.

FORMAN, D., 1991. Helicobacter pylori infection: a novel risk factor in the etiology of gastric cancer. Journal of the National Cancer Institute, 83:17021703.

FRASER, P.; CHILVERS, C.; BERAL, V. \& HILL, J. M., 1980. Nitrate and human cancer: a review of the evidence. International Journal of Epidemiology, 9:3-11.

GOODM AN, K. J. \& CORREA, P., 1995. The trasnmission of Helicobacter pylori. A critical review of the evidence. International Journal of Epidemiology, 24:875-887.

GRAHAM, S., 1984. Epidemiology of retinoids and cancer. Journal of the National Cancer Institute, 73:1423-1428.

HAENSZEL, W., 1961. Cancer mortality among the foreign born in the US. Journal of the National Cancer Institute, 26:37-132.

HAENSZEL, W. \& KURIHARA, M., 1968. Studies of japanese migrants: mortality from cancer and others diseases among japanese in the U.S. Journal of the National Cancer Institute, 40:43-68. 
HAENSZEL, W.; KURIHARA, M. \& SEGI, M., 1972. Stomach cancer among japanese in Hawaii. Journal of the National Cancer Institute, 49:969-988.

HAENSZEL, W.; KURIHARA, M.; LOCKE, F. B.; SHINUZU, K. \& SEGI, M., 1976. Stomach cancer in Japan. Journal of the National Cancer Institute, 56:265-274.

HANSSON, L. E.; ENGSTRAND, L.; NYRÉN, O.; EVANS, D. J.; LINDGREN, A.; BERGSTROM, R.; ANDERSON, B.; ATHLIN, L.; BENDTSEN, O. \&TRACZ, P., 1993. Helicobacter pylori infection: independent risk indicator of gastric adenocarcinoma. Gastroenterology, 105:1098-1103.

HOEY, J.; MONTVERNAY, C. \& LAMBERT, R., 1981. Wine and tobacco: risk factors for gastric cancer in France. American Journal of Epidemiology, 113:668-674.

HOWSON, P. C.; HYIAM A, T. \& WYNDER, L. E., 1986. The decline in gastric cancer: epidemiology of an unplanned triumph. Epidemiology Reviews, 8:127.

HU, J.; ZHANG, S.; JIA, E.; WANG, Q.; LIU, S.; LIU, Y.; WU, Y. \& CHENG, Y., 1988. Diet and Cancer of the Stomach: a case-control study in China. International Journal of Cancer, 41:331-335.

JEDRYCHOWSKI, W.; WAHRENDORF, J.; POPIELA, T. \& RACHTAN, J., 1986. A case-control study of dietary factors and stomach cancer risk in Poland. International Journal of Cancer, 37:837-842.

KNELLER, R. W.; MCLAUGHLIN, J. K. \& BJELKE, E., 1991. A cohort study of stomach cancer in a high risk american population. Cancer, 68:672-678.

KODAMA, M.; KODAMA, T.; SUSUKI, H. \& KONDO, K., 1984. Effect of rice and salty rice diet on the structure of mouse stomach. Nutrition Cancer, 6:135-147.

KOLONEL, L. N.; NOMURA, A. M. Y.; HIROHATA, T.; HANKIN, J. H. \& HINDS, M. W., 1981. Association of diet and place of birth with stomach cancer incidence in Hawaii japanese and caucasians. American Journal of Clinical Nutrition, 34:24782485.

KOLONEL, L. N.; NOMURA, A. M. Y.; HINDS, M. W.; HANKIN, J. H.; HIRIHATA, T. \& LEE, J., 1983. Role of diet in Cancer Incidence in Hawaii. Cancer Research, 43:2397s-2402s.

KONO, S. \& HIRIHATA, T., 1996. Nutrition and stomach cancer. Cancer Causes and Control, 7:41-55.

LATORRE, M. R. A mortalidade por câncer de estômago no Brasil: análise do período de 1978 a 1989. Cadernos de SaúdePública, 13(Supl. 1):67-78.

LAUREN, P., 1965. The two histological main types of gastric carcinoma: diffuse and so-called intestinal type carcinoma: an attempt at a histoclinical classification. Acta Pathologica Microbiology Scandinavian, 64:31-49.

LAVECCHIA, C.; NEGRI, E.; DECARLI, A.; D' ÁVANZO, B. \& FRANCESCHI, S., 1987. A case-control study of diet and gastric cancer in northern Italy. International Journal of Cancer, 40:484-489.

MARSHALL, B. J., 1994. Helicobacter pylori. American Journal of Gastroenterology, 89:116s-128s.

MCMICHAEL, A. J.; MCCALL, M. G. \& HARSTSHORNE, J. M., 1980. Patterns of gastro-intestinal cancer in European migrants to Australia: the role of dietary change. International Journal of Cancer, 25:431-437.

MEGRAUD, F.; RABBÉ, M. P. B.; DENIS, F.; BELBOURI, A. \& HOA, D. Q., 1989. Seroepidemiology of Campylobacter pylori infection in various populations. Journal of Clinical Microbiology, 27:1870-1873.

MIKI, K.; ICHINOSE, M.; ISHIKAWA, K. B.; YAHAGI, N.; MASASHI, M.; KAKEI, N.;TSUKUDA, S.; KIDO, M.; ISHIHAMA, S.; SHIMIZU, Y.; SUZUKI, T. \& KUROKAWA, K., 1993. Clinical application of serum pepsinogen I and II levels for mass screening to detect gastric cancer. Japanese Journal of Cancer Research, 84:1086-1090.

MILLER, A. B., 1982. Risk factors from geographic epidemiology for gastrointestinal cancer. Cancer, 50:2533-2540.

MIRVISH, S. S.; WALLCAVE, L.; EAGEN, M. \& SCHUBILE, P., 1972. Ascorbate nitrite reaction:possible means of blocking the formation of carcinogenic $\mathrm{N}$-nitroso compounds. Science, 177:65-68.

MIRVISH, S. S., 1983. The etiology of gastric cancer. Intragastric nitrosamide formation and others theories. Journal of the National Cancer Institute, 71:629-647.

MIRVISH, S. S., 1994. Experimental evidence for inhibition of $\mathrm{N}$-Nitroso compound formation as a factor in the negative correlation between vitamin C consumption and the incidence of certain cancers. Cancer Research, 54:1948s-1951s.

MS (MINISTÉRIO DA SAÚDE), 1995. Câncer no Brasil: Dados dos Registros de Base Populacional. Vol. 2, Rio de Janeiro: MS.

MUÑOZ, N., 1994. Is Helicobacter pylori a cause of gastric cancer? An appraisal of the seroepidemiological evidence. Cancer Epidemiololy, Biomarkers and Prevention, 3:445-451.

NIGHTINGALE, T. E, \& GRUBER, J., 1994. Helicobacter and human cancer. Journal of the National Cancer Institute, 86:1505-1509.

NOMURA, A.; GROVE, J. S.; STEM MERM ANN, G. N. \& SEVERSON, R. K., 1990. A prospective study of stomach cancer and its relation to diet, cigarettes and alcohol consumption. Cancer Research, 50: 627-631.

NOMURA, A.; STEMMERM ANN, G. N.; CHYOU, P. H.; KATO, I.; PEREZ-PEREZ, I.; BLASER, M . J ., 1991. Helicobacter pylori infection and gastric carcinoma among japonese americans in Hawaii. New England Journal of Medicine, 325:1132-1136.

OHGAKI, H. \& KATO, T., 1984. Study of promoting effect of sodium chloride on gastric carcinogenesis by $\mathrm{N}$-methyl- $\mathrm{N}$-nitro- $\mathrm{N}$-nitroso guanidine in inbred wistor rats. Gann, 75:1053-1057.

OHSHIMA, H. \& BARSTCH, H., 1981. Quantitative estimation of endogenous nitrosation in humans by monitoring $\mathrm{N}$-nitrosoproline excreted in the urine. Cancer Research, 41:3658-3662.

PARSONNET, J.; FRIEDM AN, G. D.; VANDERSTEEN, D. P.; CHANG, Y.; VOGELM AN, J. H.; ORENTREICH, N. \& SIBLEY, R. K., 1991. Heli cobacter pylori infection and risk of gastric carcinoma. New England Journal of Medicine, 325:1127-1131.

RISCH, A. H.; JAIN, M.; CHOI, N. W.; FODOR, G.; PFEIFFER, C. J.; HOWE, G.; HARRISON, L. W.; CRAIB, K. J. P. \& MILLER, A. B., 1985. Dietary fac- 
tors and the incidence of cancer of the stomach. American Journal of Epidemiology, 122:947-957.

ROSEN WAIKE, I., 1984. Cancer mortality among Puerto-Rican born residents in New York city. American Journal of Epidemiology, 119:177-185.

SANDER, J. \& SCHWEINSBERG, F., 1972. Wechselbeziehungen zwischen nitrat, nitrit und kanzerogenen $\mathrm{N}$-nitrosoverbindungen. Zentralblatt Fur Bakteriologie Mikrobiologie und Hygiene, 156: 299-340.

SOBALA, G. M.; SCHORAH, C. J.; SANDERSON, M.; DIXON, M. F.; TOMPKINS, D. S.; GODWIN, P. \& AXON, A. T. R., 1989. Ascorbic acid in the human stomach. Gastroenterology, 97:357-363.

SOBALA, G. M., 1991. Acute Helicobacter pylori infection: clinical features, local and systemic immune response, gastric mucosal histology, and gastric juice ascorbic acid concentrations. Gut, 32:14151418.

STEHR, P.; GLONINGER, M. F.; KULLER, L. H.; MARSH, G. M.; RADFORD, E. P. \& WEINBERG, G. B., 1985. Dietary vitamin A deficiencies and stomach cancer. American Journal of Epidemiology, 121:65-70.

TAKAHASHI, M.; KOKUBO, T. \& FURUKAWA, F., 1984. Effects of sodium chloride, saccharin, phenobarbital and aspirin on gastric carcinogenesis rats after inibition with $\mathrm{N}$-methyl-N-nitro- $\mathrm{N}$-nitroso guanidine. Gann, 75:494-501.
TATEMATSU, M.; TAKAHASHI, M . \& FUKUSHIMA, S.; 1975. Effects in rats of sodium chloride on experimental gastric cancer induced by $\mathrm{N}$-methyl $\mathrm{N}$-nitro- $\mathrm{N}$-nitrosoguanidine or 4-nitroquinoline 1-oxide. Journal of the National Cancer Institute, 55:101-106.

TANNENBAUM, S. R.; MORAN, D.; RAND, W.; CUELLO, C. \& CORREA, P., 1979. Gastric cancer in Colombia. Nitrite and other ions in gastric contents of residents from a high risk region. Journal of the National Cancer Institute, 62:9-12.

TAYLOR, D. N. \& BLASER, M. J., 1991. The epidemiology of Helicobacter pylori infection. Epidemiology Reviews, 13:42-59.

WARREN, J. R. \& MARSHALL, B. J., 1983. Unindentified curved bacilli on gastric epithelium in active chronic gastritis. Lancet, 2:1273-1275.

WEISBURGER, J. H., 1985a. Causes of gastric and esophageal cancer: possible approach to prevention by vitamin C. International Journal of Vitamin and Nutrition Research, 27:381s-402s.

WEISBURGER, J. H., 1985b. Nutrition and cancer prevention: gastrointestinal cancer. Gann Monography, 31:275-283. 